EPJ manuscript No.

(will be inserted by the editor)

\title{
Microscopic models for exotic nuclei
}

\author{
Michael Bender ${ }^{1 \mathrm{a}}$ and Paul-Henri Heenen ${ }^{2}$ \\ 1 Physics Division, Argonne National Laboratory, Argonne, Illinois 60439, U.S.A. \\ 2 Service de Physique Nucléaire Théorique, Université Libre de Bruxelles, CP 229, B-1050 Brussels, Belgium
}

February 18, 2005

\begin{abstract}
Starting from successful self-consistent mean-field models, this paper discusses why and how to go beyond the mean field approximation. To include long-range correlations from fluctuations in collective degrees of freedom, one has to consider symmetry restoration and configuration mixing, which give access to ground-state correlations and spectroscopy.
\end{abstract}

PACS. 21.60.-n Nuclear structure models and methods - 21.60.Jz Hartree-Fock and random-phase approximations - 21.10.-k Properties of nuclei; nuclear energy levels - 21.10.Dr Binding energies and masses

\section{Self-Consistent Mean-Field Models}

Self-consistent mean-field models are one of the standard approaches in nuclear structure theory, see Ref. [1] for a recent review. For heavy nuclei, they are the only fully microscopic method that can be applied systematically.

\subsection{Ingredients}

There are three basic ingredients of self-consistent meanfield models: (see Ref. 1] for references)

1.) the many-body state is assumed to be an independent-quasi-particle state of the BCS type. Degrees of freedom are a set of orthonormal single-particle states $\phi_{k}$ with corresponding operators $\hat{a}_{k}$ and occupation amplitudes $v_{k}$. The generalized one-body density matrix is idempotent

$$
\mathcal{R}^{2}=\mathcal{R}=\left(\begin{array}{cc}
\rho & \kappa \\
-\kappa^{*} & 1-\rho^{*}
\end{array}\right)=\left(\begin{array}{cc}
\left\langle\hat{a}^{\dagger} \hat{a}\right\rangle & \langle\hat{a} \hat{a}\rangle \\
\left\langle\hat{a}^{\dagger} \hat{a}^{\dagger}\right\rangle & \left\langle\hat{a} \hat{a}^{\dagger}\right\rangle
\end{array}\right) .
$$

2.) An effective interaction tailored for the purpose of mean-field calculations has to be used. It incorporates the short-range correlations induced by the strong interaction. The actually used effective interactions are parametrized and adjusted phenomenologically. They are formulated either as a density-dependent two-body force or as an energy functional $\mathcal{E}$ depending on the density matrix in the spirit of density functional theory.

3.) The equations-of-motion for the single-particle states and the occupation amplitudes are determined self-consistently from the variation of the total energy adding constraints on the particle number

$$
\delta\left(\mathcal{E}-\lambda_{N}\langle\hat{N}\rangle-\lambda_{Z}\langle\hat{Z}\rangle+\cdots\right)=0 .
$$

\footnotetext{
a Conference presenter
}

This leads to the Hartree-Fock-Bogoliubov (HFB) equations, or, using a common approximation, the HF+BCS equations. The Lagrange parameters $\lambda_{i}$ are adjusted to meet conditions for the constraint, for example $\langle\hat{N}\rangle=N$ for the average neutron number. The main ingredients of the HFB equations are the single-particle Hamiltonian and the pairing field, which are obtained as first functional derivatives of the total energy

$$
\hat{h}=\frac{\delta \mathcal{E}}{\delta \rho}, \quad \hat{\Delta}=\frac{\delta \mathcal{E}}{\delta \kappa^{*}} .
$$

\subsection{Typical Applications}

Mean-field models can be used to describe a manifold of phenomena and experimental data:

- Nuclear masses or binding energies, and all difference quantities derived from them, like one- and two-particleseparation energies, $Q$ values for $\alpha$ and $\beta$-decay.

- Deformation energy surfaces can be mapped by adding one or more constraints on a multipole moment $-\lambda_{\ell m}\left\langle\hat{Q}_{\ell m}\right\rangle$ to the variational equation (2).

- the radial density distribution and quantities derived from it as the mean-square radii of the charge and neutron distributions, the neutron skin, the surface thickness, or the full charge form factor at low momentum transfer.

- the spatial density distribution, for example multipole moments of well-deformed nuclei.

- The very concept of a single-particle energy, associated with the eigenvalues of the single-particle Hamiltonian $\hat{h}$, Eq. (3), refers to an underlying mean-field picture of the nucleus. Experimental single-particle energies, however, are obtained as an energy difference between the groundstate of an even-even nucleus on one the hand and states in adjacent odd- $A$ nuclei on the other, the latter having 


\begin{tabular}{lll}
\hline symmetry & generator & which states \\
\hline$U(1)$ gauge & particle number & pairing \\
translational & momentum & finite nuclei \\
rotational & angular momentum & deformation \\
parity & parity & octupole deformation \\
\hline
\end{tabular}

Table 1. Examples for symmetries broken in the intrinsic frame of the nucleus.

a different structure due to the unpaired nucleon, which adds significant corrections.

- Rotational bands of well-deformed nuclei can be obtained by adding a constraint on one component of the angular momentum $-\omega_{i}\left\langle\hat{J}_{i}\right\rangle$ to the variational equation (2), which is equivalent to solving the mean-field equations in a rotating frame. This adds inertial forces to the modeling, which align the angular momenta of the singleparticle states and weaken pairing with increasing total angular momentum.

\subsection{Prospects}

- Mean-field models offer an intuitive interpretation of their results in terms of the shapes of a nuclear liquid and of shells with single-particle states.

- The full model space of occupied states can be used, removing any distinction between core and valence particles and the need for effective charges.

- This allows the use of a universal effective interaction, universal in the sense that it can be applied for all nuclei throughout the periodic chart. There is, however, no consensus among practitioners of the field about a unique effective interaction. Many different functional forms have been proposed - for example non-relativistic Skyrme and Gogny interactions and finite-range as well as point-coupling relativistic interactions - and parameterizations thereof to be found in the literature [1].

\subsection{Difficulties and Problems}

- An independent particle-description establishes a bodyfixed intrinsic frame of the nucleus. The connection of mean-field results to spectroscopic observables in the laboratory frame of reference relies on additional assumptions like the rigid-rotor model, which are not valid, for example, at small deformation or in soft nuclei.

- By construction, a mean-field state breaks symmetries in the laboratory frame. Examples are given in Table 1 On the one hand, symmetry breaking is a desired feature of mean-field models. In the language of the spherical shell model (using a spherically symmetric Slater determinant as reference state) the symmetry-breaking in mean-field models adds the most important $n$-particle- $n$ hole and particle-particle correlations to the modeling at very moderate computational cost. On the other hand, a broken symmetry mixes excitations related to the symmetry operator into the mean-field state. For example, broken rotational symmetry mixes states with different values of $J^{2}$, i.e. the members of a rotational band. Broken parity mixes states of opposite parity, broken translational symmetry admixes states with different center-of-mass motion. Restoring the symmetries decomposes the mean-field states into states with proper quantum numbers.

- The mean-field approach becomes ill-defined when the binding energy changes slowly with a collective degree of freedom. This is a common situation in transitional nuclei. - It is tempting to associate two or more local minima in the potential energy landscape that are separated by a substantial barrier with different physical states, so-called shape coexistence. This interpretation might not always be valid as two different mean-field states are not orthogonal, and they might well be coupled by the interaction.

\section{Going beyond the mean field}

The idea is to start from self-consistent mean-field models as described above, keeping their advantages and successes, and to resolve the remaining problems in an efficient, systematic and consistent manner. This will add long-range correlations to the model, where "long-range" does not refer to the range of an interaction, but to collective correlations that involve the nucleus as a whole.

Two kinds of correlations have to be distinguished. As outlined above, a mean-field state describes static correlations related to deformation or pairing. These have to be distinguished from the dynamical correlations we will discuss below. They are also related to deformation and pairing, but describe fluctuations in collective degrees of freedom. The dynamical correlations cannot be described by a state for which $\mathcal{R}^{2}=\mathcal{R}$ holds and therefore require to go beyond the mean field.

\subsection{Projection Methods}

As a first-order approximation to projection, corrections to the energy are used in self-consistent mean-field models. Most prominent examples are the center-of-mass correction and the Lipkin-Nogami scheme to calculate the occupation amplitudes. Both are approximations to projection before variation (on zero momentum and particle number, respectively), when consistently included in the variation, Eq. (2). Sometimes a rotational correction to the binding energy is also applied. The corrections work best when the symmetry breaking is large, which is often not the case. It is, therefore, desirable to restore broken symmetries of the mean-field states exactly by projecting on good quantum numbers after variation. The projection might be combined with some of the correction schemes.

Eigenstates of the particle-number operator $\hat{N}$, with eigenvalue $N_{0}$, are obtained applying the particle-number projection operator

$$
\hat{P}_{N_{0}}=\frac{1}{2 \pi} \int_{0}^{2 \pi} d \phi_{N} e^{i \phi_{N}\left(\hat{N}-N_{0}\right)},
$$


while eigenstates of the angular momentum operators $\hat{J}^{2}$ and $\hat{J}_{z}$, with eigenvalues $\hbar^{2} J(J+1)$ and $\hbar M$, are obtained applying the operator

$$
\hat{P}_{M K}^{J}=\frac{2 J+1}{16 \pi^{2}} \int_{0}^{4 \pi} d \alpha \int_{0}^{\pi} d \beta \sin (\beta) \int_{0}^{2 \pi} d \gamma \mathcal{D}_{M K}^{* J} \hat{R},
$$

where $\hat{R}=e^{-i \alpha \hat{J}_{z}} e^{-i \beta \hat{J}_{y}} e^{-i \gamma \hat{J}_{z}}$ is the rotation operator and $\mathcal{D}_{M K}^{* J}$ a Wigner function. Both depend on the Euler angles $\alpha, \beta, \gamma . \hat{P}_{M K}^{J}$ picks the component with angular momentum projection $K$ along the intrinsic $z$ axis. The projected state is then obtained by summing over all $K$ components with weights determined from a variational equation. Note that $\hat{P}_{M K}^{J}$ is not a projection operator in the strict mathematical sense [2].

In the current implementation of our model, we start with $\mathrm{HF}+\mathrm{BCS}$ or HFB states $|q\rangle$ for which we assume even particle numbers, good parity, axial and time-reversal symmetry. This allows for the analytical evaluation of the $\alpha$ and $\gamma$ integration in Eq. (5) at the price of restricting the projected states to positive parity $P=+1$, even integer total angular momentum $J$, and intrinsic angular momentum projection $K=0$

$$
|J M q\rangle=\frac{\hat{P}_{M 0}^{J} \hat{P}_{N_{0}} \hat{P}_{Z_{0}}|q\rangle}{\left\langle q\left|\hat{P}_{00}^{J} \hat{P}_{N_{0}} \hat{P}_{Z_{0}}\right| q\right\rangle^{1 / 2}} .
$$

As an example, the upper left panel of Fig. [shows the decomposition of the particle-number projected energy curve for ${ }^{188} \mathrm{~Pb}$ (thin solid line) into its angular-momentum components (thick curves). All energies are normalized to the spherical state. The intrinsic spherical state is a pure $J=0$ state by construction. The difference between the meanfield and projected $J=0$ states is the rotational energy. It increases rapidly to about $3 \mathrm{MeV}$ at small $\beta_{2}$, and then grows at a slower rate with deformation.

The example of ${ }^{188} \mathrm{~Pb}$ demonstrates that projection after variation of the mean-field ground-state might not lead to the lowest projected state as it is not a variational procedure. Instead, one has to consider a set of states with different deformations and search for the energy minimum. In particular, the lowest projected state of a nucleus with a spherical mean-field ground-state is usually obtained from a deformed state. For such nuclei, there is the additional peculiarity that one obtains two $J=0$ minima at small oblate and prolate deformation, see Fig. 10 Closer examination reveals that they represent the same state, as their overlap is very close to one.

\subsection{Variational Configuration-Mixing}

The ambiguities of many near-degenerated states with different deformation can be overcome by diagonalizing the Hamiltonian in the space of these states within the Generator Coordinate Method (GCM). The mixed projected many-body state is set-up as a coherent superposition of projected mean-field states $|J M q\rangle$ with different intrinsic

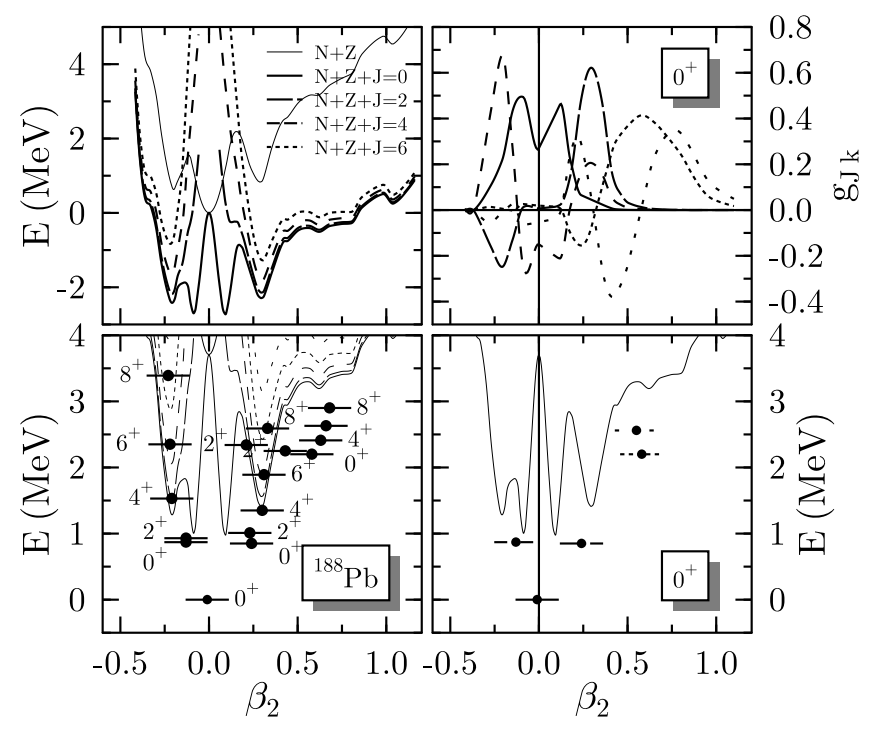

Fig. 1. Decomposition of the energy into angular-momentum components (upper left), collective wave function (upper right) and energy (lower right) for the mixed $J=0$ states, and complete spectrum of low- $J$ states (lower left) for ${ }^{188} \mathrm{~Pb}$. All curves are plotted against the mass quadrupole deformation $\beta_{2}$ of the unprojected mean-field states.

deformations $q$

$$
|J M k\rangle=\sum_{q} f_{J k}(q)|J M q\rangle,
$$

where $f_{J, k}(q)$ is a weight function which is determined from the stationarity of the states

$$
\frac{\delta}{\delta f_{J k}^{*}} \frac{\langle J M k|\hat{H}| J M k\rangle}{\langle J M k \mid J M k\rangle}=0
$$

which leads to the Hill-Wheeler-Griffin equation 3 .

$$
\sum_{q^{\prime}}\left[\left\langle J M q|\hat{H}| J M q^{\prime}\right\rangle-E_{k}\left\langle J M q \mid J M q^{\prime}\right\rangle\right] f_{J, k}\left(q^{\prime}\right)=0,
$$

that gives a correlated ground state for each value of $J$, and, in addition, a spectrum of excited states. The weight functions $f_{J k}(q)$ are not orthonormal. A set of orthonormal collective wave functions $g_{J k}(q)=\langle J M k \mid q\rangle$ in the basis of the intrinsic states is obtained from a transformation involving the square root of the norm kernel.

The actual choice for the generator coordinate depends on the mode to be described, for example, the quadrupole or octupole moment of the mass density, or the monopole moment of the pair density, which then delivers a description of quadrupole, octupole or pairing vibrations, respectively. Examples for such calculations, without angularmomentum projection, can be found in Ref. 4]. Several generator coordinates can be easily combined for multidimensional calculations, although this has been rarely done so far. For all results shown here, the axial quadrupole moment of the mass distribution serves as the generator coordinate. Hence, the excited states are either quadrupole 


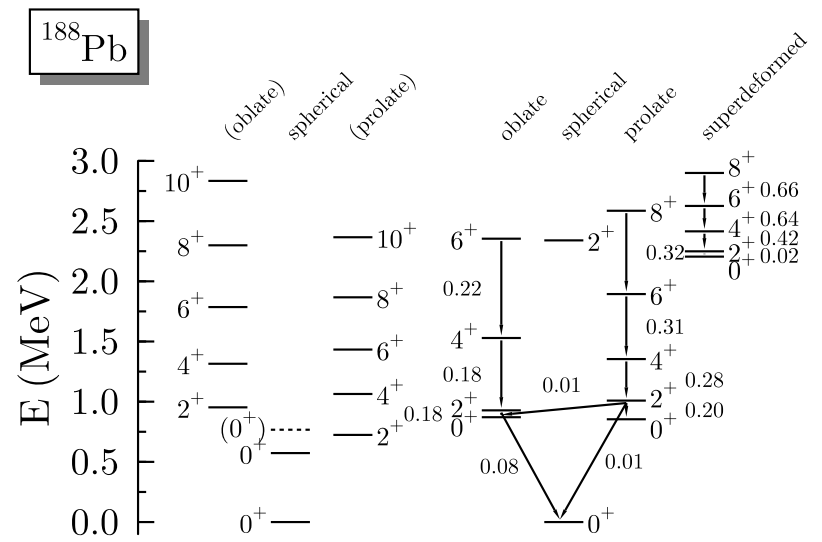

Fig. 2. Experimental (left) and calculated (right) excitation spectra and selected transition quadrupole moments $\beta_{2}^{(t)}\left(J_{k^{\prime}}^{\prime} \rightarrow J_{k}\right)$ for ${ }^{188} \mathrm{~Pb}$.

vibrational or rotational states. The right panels of Fig. 1 show as an example the mixing of the $J=0$ states with different quadrupole moments in ${ }^{188} \mathrm{~Pb}$. The upper right panel shows the collective wave functions $g_{0 k}(q)$ for the five lowest collective $J=0$ states, the lower right panel the corresponding energies drawn in the same line style by horizontal lines centered at the average deformation of the mean-field states they are composed of together with the projected $J=0$ energy curve. The energies are now normalized to the projected GCM ground state. Combining such calculations for all values of $J$ gives then the the entire spectrum of low- $J$ states, most of which can be clearly grouped into rotational bands, see the lower left panel of Fig. [1]

The projected energy curves in Fig. 1 1 are the diagonal matrix elements entering the Hill-Wheeler equation. They should not be confused with a collective potential, which does not exist in the GCM framework - nor does a collective mass. Both appear in approximations like the Bohr-Hamiltonian 5 6]. Owing to the energy gain from configuration mixing, the GCM ground state is located below the energy curves.

Projection is a special case of the GCM, where exactly degenerate states are mixed. The generators of the group involved define the collective path, and the weight functions are determined by the restored symmetry. Angularmomentum projection is part of the quadrupole correlations, as it mixes states with different orientations of the quadrupole tensor. Therefore the GCM mixing of states with respect to the quadrupole moment should be performed together with angular-momentum projection.

For a state resulting from the mixing of different meanfield states, the mean particle number is not anymore equal to the particle number of the original mean-field states. Projection, as done here, eliminates this problem, otherwise a constraint on the particle number has to be added to the Hill-Wheeler-Griffin equation (9). We also perform an approximate particle-number projection before variation in the Lipkin-Nogami approach to ensure that pairing correlations are present in all mean-field states.
The angular-momentum projected GCM allows to calculate transition moments directly in the lab frame for in-band and out-of-band transitions, for example $B(E 2)$ values

$$
\begin{aligned}
& B\left(E 2 ; J_{k^{\prime}}^{\prime} \rightarrow J_{k}\right) \\
& =\frac{e^{2}}{2 J^{\prime}+1} \sum_{M=-J}^{+J} \sum_{M^{\prime}=-J^{\prime}}^{+J^{\prime}} \sum_{\mu=-2}^{+2}\left|\left\langle J M k\left|\hat{Q}_{2 \mu}\right| J^{\prime} M^{\prime} k^{\prime}\right\rangle\right|^{2} .
\end{aligned}
$$

The $B(E 2)$ value scales with mass and angular momentum. A more intuitive measure is the transitional quadrupole deformation obtained from the $B(E 2)$ using the static rotor model

$$
\beta_{2}^{(t)}\left(J_{k^{\prime}}^{\prime} \rightarrow J_{k}\right)=\frac{4 \pi}{3 R^{2} A} \sqrt{\frac{B\left(E 2 ; J_{k} \rightarrow J_{k}^{\prime}-2\right)}{(J 020 \mid J-20)^{2} e^{2}}}
$$

with $R=1.2 A^{1 / 3}$. The method gives also the spectroscopic quadrupole moments in the lab frame

$$
Q_{s}\left(J_{k}\right)=\left\langle J M=J k\left|\hat{Q}_{20}\right| J M=J k\right\rangle
$$

which again scale with mass and angular momentum and might be given in a more intuitive measure through a dimensionless deformation parameter

$$
\beta_{2}^{(s)}\left(J_{k}\right)=-\frac{2 J+3}{J} \sqrt{\frac{5}{16 \pi}} \frac{4 \pi}{3 R^{2} A} Q_{s}\left(J_{k}\right)
$$

again with $R=1.2 A^{1 / 3}$ and assuming axial symmetry. For a given rotational band in the rigid rotor, one has $\beta_{2}^{(t)}(J+2 \rightarrow J) \approx \beta_{2}^{(s)}(J+2) \approx \beta_{2}^{(s)}(J)$ for $J>0$. Deviations from this behavior point at a more complicated situation. An example is given in Fig. 2 For high- $J$ states, the $\beta_{2}^{(t)}$ values within a band are constant, while with the mixing of the low-lying states they are significantly decreased.

\section{Examples of Applications}

All results discussed here were obtained by configuration mixing of particle-number and angular-momentum-projected $\mathrm{HF}+\mathrm{BCS}$ states with different axial mass quadrupole moments. We chose the Skyrme interactions SLy4 or SLy6 8 for the particle-hole channel and a density-dependent delta pairing interaction ("surface pairing") [9] for the particle-particle channel. A group in Madrid uses the Gogny force in a similar model [10].

\subsection{Spectroscopy}

Results for spectroscopic observables obtained with our method for ${ }^{24} \mathrm{Mg}$ have been published in 7], for ${ }^{16} \mathrm{O}$ in 11, for ${ }^{32} \mathrm{~S},{ }^{36,38} \mathrm{Ar},{ }^{40} \mathrm{Ca}$ in [12, for ${ }^{186} \mathrm{~Pb}$ in 13, for ${ }^{182-194} \mathrm{~Pb}$ in [14, and for ${ }^{240} \mathrm{Pu}$ in [15].

The neutron-deficient $\mathrm{Pb}$ isotopes show unique spectroscopic features, which are associated with a spherical 


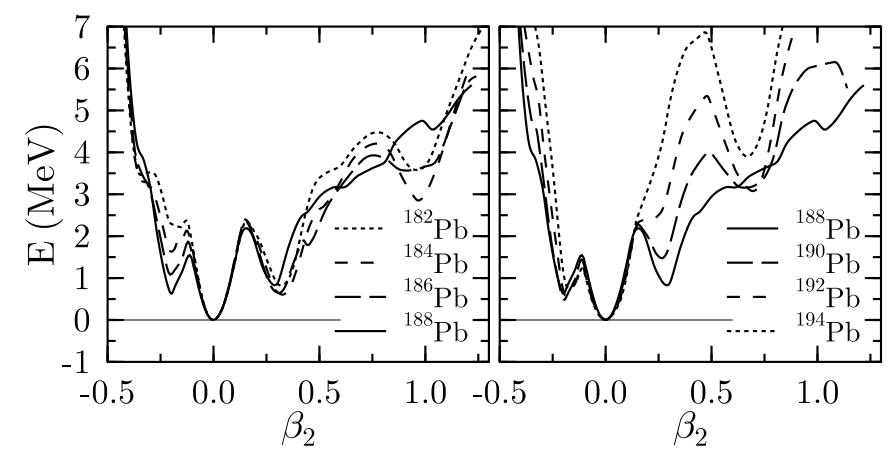

Fig. 3. Mean-field deformation energy curves for $\mathrm{Pb}$ isotopes.

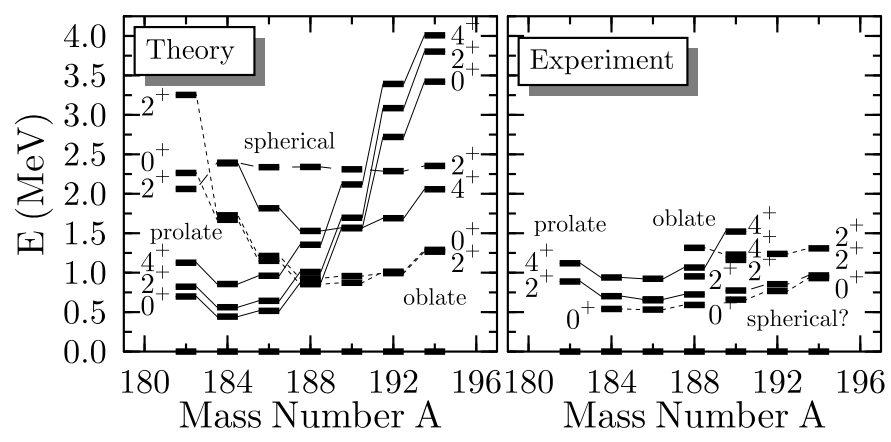

Fig. 4. Lowest collective states in the $\mathrm{Pb}$ isotopes.

ground state, an oblate minimum present above $A=188$ but disappearing below, a prolate minimum present below $A=188$ and disappearing above, and a superdeformed minimum, that is confirmed down to $A=192$, see Fig. 3. Projected GCM then delivers collective states that can be associated with a spherical ground state and excited prolate, oblate and superdeformed bands. There is a nice qualitative agreement with experimental data, see Fig. [ but the calculated transition energies within the bands are too dilute, see also Fig. 2 for ${ }^{188} \mathrm{~Pb}$. For more details and further discussion of other observables, see Refs. 13.14.

An example with very different spectroscopic features is the well-deformed nucleus ${ }^{240} \mathrm{Pu}$, see Ref. [15]. Projection does not alter the topology of the potential energy curve, but gives about $3 \mathrm{MeV}$ additional binding for the ground state and about $4 \mathrm{MeV}$ for the fission isomer which has now an excitation energy that is $1 \mathrm{MeV}$ lower compared to mean-field calculations. Projection lowers the outer barrier as much as $2 \mathrm{MeV}$. GCM does not substantially mix states. Again, the excitation energies within the rotational bands are too large, while the deformation is well described on all levels of approximation: we obtain $\beta_{2}=0.29$ for the mean-field ground state, $\beta_{2}^{(t)}(J+2 \rightarrow J)=0.30$ for all $E 2$ transitions within the ground-state band, where all excited states have spectroscopic quadrupole moments corresponding to $\beta_{2}^{(s)}(J)=$ 0.30 , in agreement with the experimental value of 0.29 deduced from the $B\left(E 2 ; 0_{1}^{+} \rightarrow 2_{1}^{+}\right)$.

\subsection{Mass Systematics}

Mass formulas based on self-consistent mean-field models using Skyrme interactions have reached a quality where they compete with the best available microscopic-macroscopic models [17. A key to this success is to add various correlation energies phenomenologically through correction terms, as a Wigner energy term or a rotational correction. There is no correction for vibrations, as it cannot be formulated in terms of a simple expression. Our model allows us to consistently calculate the quadrupole correlation energy from symmetry restoration and fluctuations of the quadrupole moment.

For the calculation of a mass table including correlations, it was necessary to use an approximation to the method described above. For this purpose, we implemented the Gaussian overlap approximation (GOA) into our method [18. While most applications of the GOA use it as an intermediate step to derive a Bohr-Hamiltonian [5] 6], we use the GOA solely as a numerical tool: a topological GOA to estimate the integrals over Euler angles from two or three exactly calculated points, and a second GOA to construct the matrices entering the Hill-Wheeler equation from diagonal matrix elements and matrix elements between nearest neighbors only. The GOA puts emphasis on the correlated $0^{+}$ground state; most information for spectroscopy is lost. Particle-number projection is still performed exactly. The accuracy of the GOA is better than $300 \mathrm{keV}$, which is sufficient for a study of the systematics of quadrupole correlation energies, which are an order of magnitude larger.

Figure 5 shows some results 19 . The overall erroneous trend with $A$, that was already observed in Ref. [20, can be removed with a slight refit of the coupling constants of SLy4 on the mean-field level [21]. The quadrupole cor-

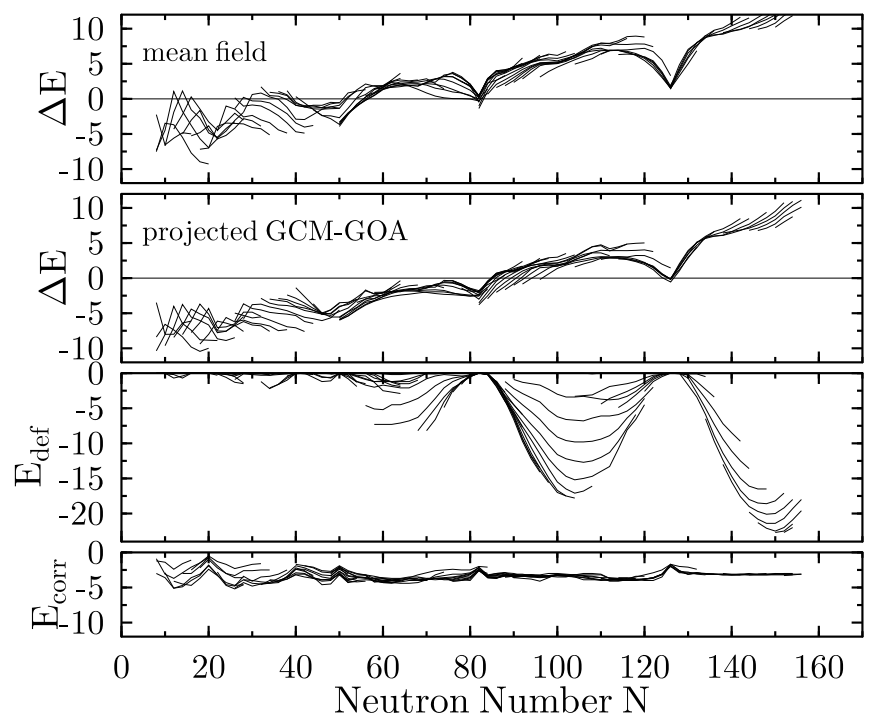

Fig. 5. Upper two panels: difference between calculated and experimental masses. Lower two panels: mean-field deformation energy $E_{\text {def }}$ and beyond-mean-field quadrupole correlation energy $E_{\text {corr }}$. All panels share the same energy scale in $\mathrm{MeV}$. 


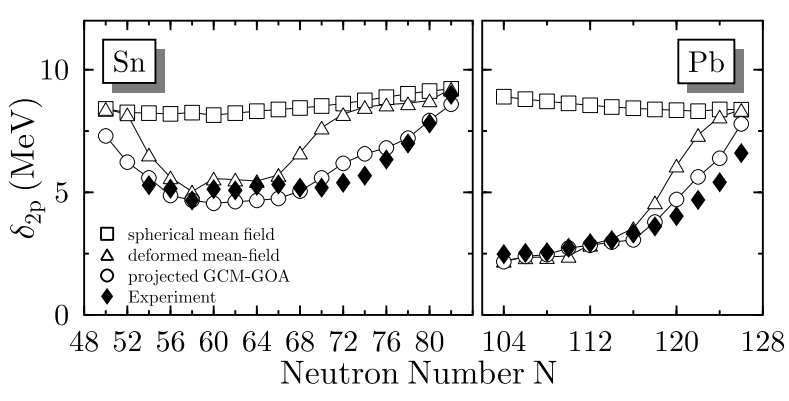

Fig. 6. Two-proton gap $\delta_{2 p}$ for $\mathrm{Sn}$ and $\mathrm{Pb}$ isotopes.

relation energy improves the masses by reducing the oscillations between the closed shells, but without removing them completely. Still, there is a substantial improvement, which becomes obvious when looking at energy differences like the two-proton gap $\delta_{2 p}(N, Z)=E(N, Z-2)-$ $2 E(N, Z)+E(N, Z+2)$, see Fig. [6] Spherical mean-field calculations (open squares) give near-constant $\delta_{2 p}$ in accordance with the stable $Z=82$ shell. Allowing for deformation (open triangles) and adding correlations (open circles) substantially reduces $\delta_{2 p}$ for mid-shell nuclei far from ${ }^{208} \mathrm{~Pb}$, in agreement with experiment (full diamonds). Similar results were obtained in Ref. 22].

The lower panels of figure 5 compare mean-field deformation and beyond-mean-field quadrupole correlation energies. While heavy nuclei are dominated by the deformation energy, light nuclei are dominated by the correlation energy.

\section{Summary and Outlook}

Projection and configuration mixing significantly improve the modeling of nuclei in self-consistent mean-field approaches and give access to spectroscopy. Masses are significantly improved around closed shells, and the overall structure of collective bands is reproduced, even in complicated systems like neutron-deficient $\mathrm{Pb}$ isotopes where many structures coexist. On the quantitative level, neither masses nor excitation spectra are yet described with the desired precision. This might be for a number of origins. There will be imperfections of the effective interactions that we use. Some aspects of the effective interaction might be much more sensitive to spectroscopy than to the ground states they are fitted to. For consistency, the effective interaction should be refitted including the correlations. To obtain a more robust extrapolation of the interaction into the unknown, it is desirable to establish a link between the effective interaction needed for calculations as done here, and more ab-initio methods.

On the other hand, the modeling of the configuration mixing might still have some deficiencies as well. There are additional modes like pairing vibrations, triaxial quadrupole deformations, or octupole vibrations, which might play a role for certain nuclei and, therefore, should be included in a unified model. The determination of the collective path has to be re-examined, and diabatic states may play a role in some situations. An interesting insight comes from self-consistent, cranked mean-field calculations: for ${ }^{240} \mathrm{Pu}$, the excitation energies from cranked HFB are in much better agreement with experiment than our projected values when using the same interaction [15]. Cranked mean-field states break time-reversal invariance and have the proper angular momentum on the average, which might be crucial for excitation energies. A generalization of our model to use cranked states as a starting point for projected GCM is currently underway. This will also allow to describe nuclei with an odd nucleon number in our framework. A lot of work is left for the future, but present results are most encouraging.

\section{Acknowledgements}

The results discussed here were obtained in collaboration with G. F. Bertsch, P. Bonche, T. Duguet, and H. Flocard. We thank T. Duguet and R. V. F. Janssens for critical reading of the manuscript. MB thanks for the warm hospitality at the Service de Physique Nucléaire Théorique at the Université Libre de Bruxelles, Belgium, and the Institute for Nuclear Theory, Seattle, USA, where parts of the research presented here were carried out. This work was supported in parts by the U.S. Department of Energy, Office of Nuclear Physics, under Grant W-31-109-ENG-38 (Argonne National Laboratory) and the Belgian Science Policy Office under contract PAI P5-07.

\section{References}

1. M. Bender, P.-H. Heenen, P.-G. Reinhard, Rev. Mod. Phys. 75, 121 (2003).

2. P. Ring, P. Schuck, The Nuclear Many-Body Problem, Springer Verlag, New York, Heidelberg, Berlin, 438 (1980).

3. D. L. Hill, J. A. Wheeler, Phys. Rev. 89, 1106 (1953); J. J. Griffin, J. A. Wheeler, Phys. Rev. 108, 311 (1957).

4. P.-H. Heenen et al., Eur. Phys. J. A11, 393 (2001) .

5. J. Libert et al., Phys. Rev. C 60, 054301 (1999).

6. P. Fleischer et al., Phys. Rev. C 70, 054321 (2004).

7. A. Valor et al., Nucl. Phys. A671, 145 (2000).

8. E. Chabanat et al., Nucl. Phys. A635, 231 (1998).

9. C. Rigollet et al., Phys. Rev. C 59, 3120 (1999).

10. R. Rodriguez-Guzman et al., Phys. Rev. C 62, 054319 (2002).

11. M. Bender, P.-H. Heenen, Nucl. Phys. A713, 390 (2003).

12. M. Bender et al., Phys. Rev. C 68, 044321 (2003).

13. T. Duguet et al., Phys. Lett. B559, 201 (2003).

14. M. Bender et al., Phys. Rev. C 69, 064303 (2004).

15. M. Bender et al., Phys. Rev. C 70, 054304 (2004).

16. M. Bender et al., Eur. Phys. J. A7, 467 (2003).

17. M. Samyn et al., Nucl. Phys. A700, 142 (2002); Phys. Rev. C 70, 044309 (2004).

18. M. Bender et al., Phys. Rev. C 69, 034340 (2004).

19. M. Bender et al., nucl-th/0410023

20. M. V. Stoitsov et al., Phys. Rev. C 68, 054312 (2003).

21. G. F. Bertsch et al., preprint nucl-th/0412091

22. P. Fleischer et al., Eur. Phys. J. A22, 363 (2004). 